\title{
Rareté de ressources et conflit entre pasteurs et agriculteurs au Sud-Kordofan, Soudan
}

Les obstacles à la promotion du pastoralisme comme forme d'adaptation au changement climatique

Resource scarcity and pastoral-farmer conflict in Southern Kordofan, Sudan: hurdles for the promotion of pastoralism as a form of adaptation to climate change

Salomé Bronkhorst

\section{(2) OpenEdition}

Journals

Édition électronique

URL : http://journals.openedition.org/conflits/18589

DOI : $10.4000 /$ conflits. 18589

ISSN : $1777-5345$

Éditeur :

CCLS - Centre d'études sur les conflits lilberté et sécurité, L'Harmattan

Édition imprimée

Date de publication : 30 décembre 2012

Pagination : 111-132

ISBN : 978-2-343-00589-8

ISSN : 1157-996X

Référence électronique

Salomé Bronkhorst, "Rareté de ressources et conflit entre pasteurs et agriculteurs au Sud-

Kordofan, Soudan », Cultures \& Conflits [En ligne], 88 | hiver 2012, mis en ligne le 15 mars 2014,

consulté le 30 mars 2021. URL : http://journals.openedition.org/conflits/18589 ; DOI : https://doi.org/ 10.4000/conflits.18589 


\title{
Rareté de ressources et conflit entre pasteurs et agriculteurs au Sud-Kordofan, Soudan
}

\author{
Les obstacles à la promotion du pastoralisme \\ comme forme d'adaptation au changement climatique 1
}

\section{Salomé BRONKHORST}

Salomé Bronkhorst consacre ses recherches au lien entre l'environnement, le climat, le développement et les conflits en Afrique. Elle est actuellement consultante; son affectation la plus récente étant au Centre africain pour la résolution constructive des conflits (ACCORD). Ses travaux portent sur les conflits en Afrique et le développement en liaison avec la sécurité humaine, le changement climatique, les conflits pastoraux, les conflits communautaires, et les politiques d'adaptation au changement climatique.

Tly a des preuves solides montrant que le pastoralisme ${ }^{2}$ en Afrique a émergé il y a sept mille ans et qu'il s'est progressivement étendu dans le nord de l'Afrique comme une réponse pour faire face aux incertitudes climatiques et aux sécheresses croissantes ${ }^{3}$. En fait, " la préhistoire et l'histoire sont marquées par des mouvements humains (épisodiques et localisés) d'une zone cli-

1. Texte traduit de l'anglais par Marie Georgopoulos. Toutes les traductions des citations anglaises sont du même traducteur.

2. Le pastoralisme est une forme de production des moyens de subsistance basé sur l'élevage. Les pasteurs, en fonction de l'ampleur du mouvement, peuvent être qualifiés de nomades, transhumants et d'agro-pasteurs. Les mouvements des nomades ne sont pas prédéterminés, alors que les pasteurs transhumants déplacent le bétail sur des trajectoires fixes selon les saisons. Les agro-pasteurs cultivent la terre et disposent d'un logement et d'autres infrastructures; en même temps qu'ils détiennent des troupeaux de petite taille, ils peuvent envoyer loin des troupeaux plus grands en les confiant aux pasteurs nomades. Voir : Blench R., You can't go home again: pastoralism in the new millennium, London, Institut du développement d'outre-mer, 17 mai 2001, pp. 11 et 13. Cet article s'intéresse à la transhumance et à l'agro-pastoralisme pratiqués par les groupes ethniques qui pratiquent le pastoralisme dans l'État du Sud Kordofan, au Soudan.

3. Brooks N., Climate change, drought and pastoralism in the Sabel, Note de discussion pour l'Initiative Mondiale sur le Pastoralisme Durable, WISP/IUCN, novembre 2006, p. 3. http://www.nickbrooks.org/publications/WISP_CCAP_final_en_v2.pdf (Page consultée le 19 juin 2011). 
matique à une autre, car les gens étaient continuellement à la recherche d'environnements permettant de garantir leur survie et de satisfaire leurs aspirations à une existence plus stable ${ }^{4}$ ».

Le pastoralisme africain a été souvent perçu, au début du colonialisme, comme un système de subsistance "stagnante, improductive, et écologiquement néfaste 5 », ce qui a généré, jusqu'il y a une quinzaine d'années, des politiques et des financements encourageant le sédentarisme, le déstockage des troupeaux et autres mesures visant à forcer les éleveurs à se sédentariser. Ces dernières années, cependant, un nouveau paradigme a émergé. Favorable au développement du pastoralisme, il reconnaît sa valeur pour les économies africaines et l'environnement ${ }^{6}$. Certains vont même jusqu'à affirmer que le pastoralisme doit être promu comme une forme d'adaptation proactive au changement climatique ${ }^{7}$. Par exemple, le Overseas Development Institute (ODI, un groupe de réflexion britannique) et le Humanitarian Policy Group, soutiennent que « le pastoralisme fonctionne mieux dans un contexte général de forte variabilité des précipitations et marqué par l'imprévisibilité. Avec des politiques publiques adéquates, de l'investissement et du soutien, le pastoralisme se présente comme un parcours rationnel d'adaptation dans des régions maquées par une variabilité climatique accrue, et a un rôle important à jouer là où d'autres moyens de subsistance sont susceptibles d'échouer 8 ». Bien que l'ODI reconnaisse les limites des scénarios de projections climatiques et semble largement conscient des défis auxquels les éleveurs sur le continent africain sont confrontés, il serait prudent de ne pas ignorer ceux qui expriment leur préoccupation concernant l'avenir du pastoralisme dans le continent au regard des présentes et futures vulnérabilités écologiques, sociales et politiques 9 .

En tant que forme de migration saisonnière déterminée par et dépendante de la variabilité et du changement climatique, le pastoralisme, dans la région semi-aride du Kordofan Sud au Soudan, sera - selon les projections climatiques actuelles - significativement affecté par le changement du climat 10.

4. Warner K., Hamza M., Oliver-Smith A., Renaud F., Julca, A. “Climate change, environmental degradation and migration”, Natural Hazards, vol. 55, 2010, pp. 689-715.

5. Turner M.D., “The new pastoral development paradigm: engaging the realities of property institutions and livestock mobility in dryland Africa”, Society and Natural Resources, vol. 24, 2011, pp. 469 - 484.

6. Ibid.

7. L'adaptation est ici définie comme l'ajustement des systèmes naturels ou humains à des stimuli climatiques présents ou futurs, ou à leurs impacts, afin d'atténuer leurs effets néfastes ou d'exploiter leurs effets bénéfiques (GIEC, 2001). Ci-dessous seront utilisés les termes « adaptation au changement climatique » et « adaptation ».

8. ODI (Institut du développement d'outre-mer), "Pastoralism and climate change: Enabling adaptive capacity”, Synthesis Paper, London, avril, 2009, p 1.

9. Ibid. ; Blench R., You can't go home again: pastoralism in the new millennium, op. cit. ; Casciarri B., Ahmed A.G.M., "Pastoralists under pressure in present-day Sudan: an introduction”, Nomadic Peoples, vol. 13, n 1, 2009, pp. 10-22 ; Stige L.C., Stave J., Chan K., Ciannelli L., Pettorelli N., Glantz M., Herren H.R., Stenseth N., "The effect of climate variation on agro-pastoral production in Africa”, PNAS, vol. 103, n 9, février 2006 ; Brooks N., op. cit. ; Turner, M. D., op. cit. 
Dans l'évaluation effectuée par le Fonds pour l'environnement mondial (FEM) en vue d'apprécier le financement nécessaire à l'adaptation au changement climatique au Soudan, il est indiqué que la température moyenne annuelle devrait augmenter entre 0.8 à 1.7 degrés Celsius d'ici 2030 et qu'une plus grande variabilité des précipitations est attendue, surtout pendant la saison des pluies. Les conditions propices à l'agriculture se déplaceront plus au sud, réduisant ainsi les récoltes de sorgho, de millet et de fourrage pour le bétail et laissant les agriculteurs et les pasteurs incapables de maintenir leurs moyens de subsistance ${ }^{11}$. Il est également attendu que le changement climatique affecte la disponibilité des ressources en eau avec une baisse de 0,5\% par an des précipitations calculée entre 1941 et 2000 et une plus grande variabilité des précipitations enregistrées ${ }^{12}$. Enfin, il est estimé qu'une avancée du désert, entre $50 \mathrm{~km}$ et $200 \mathrm{~km}$ vers le sud a eu lieu depuis le début des enregistrements en 1930 13. Ceci est préoccupant pour au moins deux raisons. Premièrement, le pastoralisme constitue une source essentielle de production de biens de subsistance dans le Kordofan méridional et au-delà. Bien que la cartographie des migrations pastorales et leur nombre réel soient largement conjecturels, il est estimé que la production pastorale est pratiquée sur un quart des superficies mondiales et sur $66 \%$ des superficies africaines, et représentant $20-25 \%$ du PIB agricole de l'Afrique ${ }^{14}$. L'agriculture pluviale, dépendante de la pluviométrie, et l'élevage ont été et sont les principales sources de subsistance pour les groupes ethniques et les tribus du Soudan. Au Soudan, 80 \% de la population tire une partie de ses moyens de subsistance de l'agriculture et de l'élevage, avec le pastoralisme pratiqué par plus de $20 \%$ de la population ${ }^{15}$. Deuxièmement, si le pastoralisme doit être promu comme une forme d'adaptation au changement climatique, il faudra plus de recherches sur : (a) l'incidence qu'a exactement le changement climatique sur le pastoralisme ; (b) les difficultés climatiques et non climatiques rencontrées par les éleveurs en Afrique. Notre analyse portera plus spécifiquement sur cette deuxième question et examinera les conflits entre éleveurs et agriculteurs liés à la rareté des ressources dans le Kordofan méridional.

Cet article s'appuie sur l'analyse des conflits effectuée par des chercheurs, des agences d'aide internationale, le gouvernement soudanais et des organisa-

10. Adepoju A., "Migration in Africa: an overview", Baker J., Akin Aina T. (eds), The migration experience in Africa, Uppsala, Nordic Africa Institute, 1995 ; OCDE (Organisation de la coopération et du développement économique), "Promoting and supporting change in transhumant pastoralism in the Sahel and West Africa”, Policy Note, n 3, 2007, pp. 87-108.

11. FEM, Least Developed Countries Fund: Project Identification Form for Sudan, 17 août 2007.

12. Ibid.

13. Programme des Nations Unies pour l'Environnement (PNUE), Sudan post-conflict environmental assessment, Nairobi, 2007, p. 9.

14. Blench R., op. cit. ; Nori, M., Taylor M., Sensi A., Browsing on fences: pastoral land rights, liveliboods and adaptation to climate change, International Institute for Environment and Development (IIED), London, mai 2008 ; ODI, op. cit.

15. FEM, op. cit. ; Plan des Nations Unies pour le Développement (PNUD), Pastoral production systems in Southern Kordofan, Khartoum, 2006. 
tions de la société civile qui travaillent sur le terrain pour favoriser le règlement des conflits entre éleveurs et agriculteurs du Kordofan Sud. Cette analyse est complétée par un travail de terrain réalisé en Juillet 2010 au Soudan ${ }^{16}$. La première partie porte sur les débats théoriques relatifs aux liens entre migrations, changement climatique et conflits. L'accent est plus particulièrement mis sur la thèse de la rareté des ressources. La deuxième partie examine les conflits entre éleveurs et agriculteurs dans le Kordofan méridional en les situant dans le contexte socio-économique, environnemental et politique en présence. Elle propose une typologie de ces conflits agro-pastoraux et met en avant deux facteurs qui à la fois créent, maintiennent et perpétuent la rareté des ressources et érodent les capacités des éleveurs et des agriculteurs à faire face à cette rareté ainsi qu'aux conflits qui en découlent. Ces facteurs sont, d'abord, le droit des sols et les politiques d'agriculture mécanisée et, ensuite, les politiques qui ont affaibli l'administration indigène, seule institution ayant démontré sa capacité à gérer les ressources, à faire face aux pénuries et à régler les conflits entre les groupes antagonistes. Nous tenterons de démontrer, dans ce contexte socio-économique et ethno-politique unique, qu'il s'agit des deux facteurs principaux à l'origine des conflits agro-pastoraux dans le Kordofan Sud, et de deux obstacles majeurs à la promotion du pastoralisme comme une forme d'adaptation au changement climatique dans cet État. En guise de conclusion, la troisième partie discutera des implications pour le pastoralisme dans le Kordofan méridional et pour sa promotion comme une forme d'adaptation au changement climatique au Soudan.

\section{Changement climatique, migrations et conflits : le rôle de la rareté des ressources}

Il est largement reconnu que le changement climatique aura des répercussions profondes et posera des défis pour le développement et la sécurité humaine à une échelle sans précédent, particulièrement pour l'Afrique et certaines parties du monde en voie de développement ${ }^{17}$. Mais va-t-il générer des conflits ? Bien que le débat climatique connaisse, de plus en plus, un tournant "sécuritaire ", le consensus est limité sur les liens exacts entre changement climatique et conflits ${ }^{18}$. Le rapport de la Troisième évaluation du GIEC de 2001,

16. Un rapport de recherche intitulé «Changement climatique et conflit : leçons à tirer pour la résolution des conflits du Sahel Sud au Soudan ", a été publié en 2011à partir de cette étude, par le Centre africain pour la résolution constructive des conflits (ACCORD). L'étude a été basée sur la prémisse que, pour répondre aux conflits liés au changement climatique, des leçons importantes pourraient être tirées en examinant les interventions des organisations internationales et des ONG qui ont porté sur de conflits environnementaux dans cette zone.

17. Nordås R., Gleditsch N., "Climate change and conflict”, Political Geography, vol. 26, 2007, pp. 627-638 ; GIEC, Contribution of Working Group II to the Fourth Assessment Report of the Intergovernmental Panel on Climate Change, 2007 ; Barnett J., Adger W.N., "Climate change, human security and violent conflict”, Political Geography, vol. 26, 2007, p. 561, http://www.ipcc.ch/pdf/assessment-report/ar4/wg2/ar4-wg2-spm.pdf

18. Brown O., Hammill A., McLeman R., "Climate change as the 'new' security threat: Implications for Africa”, International Affairs, vol. 83, n 6, 2007, pp. 1141-1154. 
qui souligne les impacts potentiels du changement climatique en matière de sécurité, est critiqué par ceux qui lui reprochent de s'appuyer sur des données secondaires et à caractère politique ${ }^{19}$. Toutefois, d'autres études éclairent ces liens entre climat et conflit, en examinant l'effet de variables climatiques, comme les précipitations et les températures, sur l'émergence de conflits 20. Un certain consensus semble se dessiner pour considérer que si les facteurs climatiques et environnementaux ne suffisent pas à eux seuls à générer des conflits, ils peuvent alimentent ou aggraver les origines sociales, politiques ou économiques d'un conflit ${ }^{21}$.

En ce qui concerne les migrations, les impacts prévus par le changement des températures et l'évolution des précipitations et d'autres phénomènes météorologiques peuvent améliorer les conditions de vie et de subsistance dans certaines régions et rendre d'autres inhabitables. Tandis que la plupart des ces changements seront graduels, permettant aux gens de s'adapter, certains peuvent être soudains, forçant les gens à quitter leur lieu habituel de vie. A travers l'élévation du niveau des mers, les changements météorologiques, les précipitations, et la prolifération de certains virus, les conditions de vie des populations seront affectées de diverses manières, forçant les gens à devenir mobiles ou à modifier leurs modes d'existence pastorale ${ }^{22}$. En effet, le GIEC estime que l'impact le plus dramatique du changement climatique peut concerner la migration 23.

La migration climatique est un phénomène complexe qui se situe au croisement de différents facteurs : la mesure dans laquelle la migration est volontaire ou forcée, temporaire (saisonnière) ou permanente, interne ou internationale, et le niveau de vulnérabilité ou de résilience des individus et des communautés face à un risque environnemental ou une menace ${ }^{24}$. Les chercheurs

19. Gleditsch N. P., Nordås R., Salehyan I., "Climate change, migration and conflict: the migration link”, Coping with Crisis, Working Paper Series, New York, International Peace Academy, 2007.

20. Solomon H., Turton A., (eds), Water wars: Enduring myth of impending reality, Africa Dialogue Monograph Series, $n^{\circ}$ 2., Durban, African Centre for the Constructive Resolution of Disputes, 2000 ; Hendrix C.S., Glaser S.M., "Trends and triggers: climate, climate change and civil conflict in Sub-Saharan Africa", Political Geography, vol. 26, 2007, pp. 695-715; Burke M. B., Miguel E., Satyanath S., Dykema J.A., Lobell D.B., "Warming increases the risk of civil war in Africa", Proceedings of the National Academy of Sciences, vol. 106, n 49, 2009, pp. $20670-20674$.

21. Gleditsch N.P., Regional Conflict and Climate Change, Paper for workshop on research on climate change impacts and associated economic damages, Washington DC, 2011, disponible : http://yosemite.epa.gov/ee/epa/eerm.nsf/vwAN/EE-0566-122.pdf/\$file/EE-0566122.pdf (Page consultée le 5 mai 2011) ; Conseil Consultatif Allemand sur le Changement Global (WBGU), World in transition: climate change as a security risk, London and Stirling, 2007 ; Yanda P., Bronkhorst S., "Climate change and conflict: Conflict-sensitive climate change adaptation in Africa", Policy and Practice Brief, Durban, ACCORD, 2011 ; Raleigh C., Urdal H., "Climate change, environmental degradation and armed conflict", Political Geography, vol. 26, 2007, pp. 656-673.

22. Nordås R, Gleditsch N., op. cit., p. 634.

23. GIEC, op. cit.

24. Naik A., "Migration and natural disasters", Migration, Environment and Climate Change: 
reconnaissent également les divers effets du climat sur les migrations, tels que les changements soudains (inondations ou catastrophes naturelles par exemple) et les changements progressifs, la désertification par exemple ${ }^{25}$. En cas de changement majeur et soudain, les gens peuvent être déplacés involontairement, mais une grande partie du mouvement migratoire est supposée représenter un choix raisonné ou une réponse d'adaptation aux changements environnementaux ${ }^{26}$. Dans ce cas, il est probable que des facteurs d'attraction, «pull factors ", joueront un rôle plus important dans la décision de migrer. Pour les pasteurs, il s'agira par exemple d'opportunités de pâturage ou de disponibilités en eau.

Quant aux effets du changement climatique sur les migrations saisonnières telles que le pastoralisme, des études récentes montrent que les bergers démontraient une plus forte capacité d'adaptation au changement climatique dans le passé qu'aujourd'hui, parce qu'ils font face à un certain nombre d'autres défis, non écologiques, qui affectent leur capacité à s'adapter ${ }^{27}$. Ces défis incluent la pauvreté, la marginalisation politique et économique et les politiques de développement inappropriées qui entravent la mobilité des troupeaux, affectent les droits et l'accès aux ressources, et même affaiblissent les institutions traditionnelles de gestion des conflits, qui sont essentielles pour négocier à la fois l'accès aux ressources et la résolution d'un conflit tant au Soudan qu'ailleurs. Ces défis issus de multiples domaines deviennent apparents dans les débats sur les liens entre climat et migrations où certains, qualifiés d' " alarmistes », identifient l'environnement comme le facteur causal de migration, pendant que d'autres, dits « sceptiques », insistent sur la complexité et la multitude des facteurs qui sont à l'origine de la décision de migrer ${ }^{28}$ et que d'autres enfin considèrent que les changements climatiques forment seulement une variable parmi d'autres dans la décision de migrer ${ }^{29}$.

Assessing the evidence, Geneva, Organisation internationale pour les migrations (OIM), 2009.

25. Warner K., Hamza M., Oliver-Smith A., Renaud F., Julca A., op. cit.

26. McLeman R., Smit B., "Migration as an adaptation to climate change", Climatic Change, vol. 76, 2006, pp. 31-53.

27. Neely C., Bunning, S., "Review of evidence on dryland pastoral systems and climate change: implications and opportunities for mitigation and adaptation", FAO - NRL Working Paper, Rome, 2008 ; Oxfam, Survival of the fittest: pastoralism and climate change in East Africa, Oxfam Briefing paper, $n^{\circ} 116$, août 2008 ; Hesse C., Cotula L., "Climate change and pastoralists: investing in people to respond to adversity”, Sustainable Development Opinion, International Institute for Sustainable Development, London, 2006.

28. Dun O., Gemenne F., "Defining environmental migration: Why it matters so much, why it is controversial and some practical processes which may help move forward", Forced Migration Review, vol. 31, 2008, pp. 10-11, http://www.fmreview.org/climatechange.htm. (Page consultée le 1er juin 2011); Castles S., "Environmental change and forced migration: making sense of the debate”, New Issues in Refugee Research, Working Paper, n 70, Genève, Haut Commissariat des Nations Unies pour les réfugiés (UNHCR), 2002, pp. 1-14 ; Hugo G., "Migration, development and environment", Migration Research Series, $\mathrm{n}^{\circ} 35$, Geneva, Organisation internationale pour les migrations (OIM), 2008.

29. Reuveny R., "Climate change induced migration and violent conflict", Political Geography, vol. 26, 2007, pp. 656-673 ; Dun O., Linkages between flooding, migration and resettlement in the Mekong Delta, Vietnam: Case Study Report, EACH-FOR Project, UNU-EHS, Bonn, 
Quant à la manière dont le changement climatique, les migrations et les conflits peuvent interagir entre eux, certains chercheurs spécialistes en sécurité environnementale conceptualisent les liens entre ces variables en termes de rareté et/ou abondance des ressources ${ }^{30}$. Leur thèse consiste à soutenir que, particulièrement dans les pays les moins développés où de nombreuses personnes dépendent de l'environnement pour leur subsistance, le changement climatique pourrait conduire à des conflits dans les lieux d'accueil des migrants dans la mesure où la migration accroît la densité démographique dans ces zones alors que la quantité de ressources reste stable, baisse ou se détériore ${ }^{31}$. Cette thèse nous paraît utile pour l'analyse du Kordofan Sud, du fait que le pastoralisme consiste en une forme de mobilité qui affecte non seulement les zones d'accueil, mais toutes les étapes de la route conduisant à une destination donnée. Ce pays est fortement dépendant des ressources naturelles, et, comme cela sera démontré plus loin, leur rareté joue un rôle crucial dans les conflits qui émergent entre éleveurs et agriculteurs.

Le discours sur la rareté, avec ses composantes malthusiennes, a été relancé dans les années 1990 par Robert Kaplan, alors que son ouvrage Coming Anarchy 32 était tombé en désuétude dans la décennie précédente, alors que les théories du conflit se focalisaient sur les causes ethniques et religieuses des conflits ${ }^{33}$. Ce discours porte sur un ensemble de « ressources vitales » dont une population dépend pour sa survie et son bien-être économique et sur les conditions pouvant rendre ces ressources « rares ${ }^{34}$. Trois types de développement, qui sont interconnectés, dommageables et qui se renforcent mutuellement, pourraient conduire à la raréfaction d'une ressource ${ }^{35}$ :

- un développement qui s'affaiblit qualitativement jusqu'à s'épuiser ou se dégrader entraînant ainsi une réduction du « gâteau » disponible pour tous.

- Un développement dont la disponibilité diminue, entraînant une baisse

2009 ; Black R, "Environmental refugees: myth or reality?”, New issues in refugee research, Working paper, $\mathrm{n}^{\circ} 34$, Genève, Haut Commissariat des Nations Unies pour les réfugiés (UNHCR), 2001.

30. McLeman R., "Climate change, migration and critical international security considerations", Migration Research Series, Rapport n ${ }^{\circ} 42$, Genève, Haut Commissariat des Nations Unies pour les Réfugiés (UNHCR), 2011 ; Homer-Dixon T., "On the threshold: environmental changes as causes of acute conflict", International Security, vol. 16, n 2, 1991, pp. 76-116; Homer-Dixon T., "Environmental Scarcity and Mass Violence", in Gearoid O., Tuathail, S. D., Routledge P., (eds), The Geopolitics Reader, London, Routledge, 1998, pp. 204-211 (réimprimé par Current History, 1996); Homer-Dixon T. F., Environment, scarcity, and violence, Princeton, Princeton University Press, 1999.

31. On peut soutenir que dans le cas des migrations saisonnières, « les zones d'accueil » incluraient également les zones de transit où les pasteurs font paître et abreuvent leur bétail.

32. Pour d'autres études néo-malthusiennes, voir Hardin G., "The tragedy of the commons", Science, $\mathrm{n}^{\circ}$ 162, 1968, pp. 1243-1248 et Ehrlich P., Ehrlich A., Betrayal of Science and Reason: How Anti-Environmental rhetoric threatens our future, Washington, DC, Island, 1996.

33. Kaplan R. D., "The coming anarchy", The Atlantic Monthly, 1er février 1994.

34. McLeman R, 2011, op. cit.

35. Homer-Dixon T., 1998, op. cit. ; McLeman R., op. cit. 
des parts revenant à chaque citoyen ou groupe. Un exemple pourrait être une augmentation de la population dépendante de la même ressource.

- Un développement qui devient moins accessible pour certains, lorsque tous ne reçoivent plus une part égale $d u$ " gâteau ». Homer Dixon a qualifié ce phénomène de rareté structurelle. Un exemple pourrait être les limites imposées par certains sur une ressource, pour en exclure d'autres ${ }^{36}$.

Le Projet Changement Environnemental et Conflit Aigu (Environmental Change and Acute Conflict Project ECACP) sous la direction de Thomas Homer-Dixon, qui a réuni une centaine d'experts venus du monde entier, rassemble quatorze études de cas de rareté environnementale, notamment au Rwanda, en Chine, en Inde, et en Afrique du Sud. Le projet aboutit à la conclusion que de graves situations de rareté environnementale génèrent un effondrement social et des situations de violence. Les dynamiques en ouvre sont complexes, mais peuvent être présentées comme suit : la rareté des ressources réduit la production alimentaire, appauvrit les gens déjà pauvres, et peut pousser à la migration ; parallèlement, le contrôle imposé sur certaines ressources peut enrichir les élites et miner l'autorité morale d'un État et sa capacité à gouverner. Ces tensions et mouvements de long terme peuvent déchirer progressivement le tissu social d'une société pauvre, provoquer des révoltes populaires chroniques et de la violence, tout en augmentant les inégalités et en modifiant le rapport de forces entre groupes sociaux antagonistes et l'État ${ }^{37}$. Dans le cas du Kordofan méridional, les dynamiques en jeu paraissent tout aussi complexes, avec l'émergence de situations de rareté qui suscitent des réactions spécifiques de la part des gens, en fonction du contexte socio-économique, ethno-politique et historique en présence.

\section{Conflits entre pasteurs et agriculteurs dans le sud du Kordofan et rareté des ressources}

\section{Contexte socio-économique et environnemental}

Les éleveurs dans le Kordofan méridional vivent essentiellement de l'élevage du bétail dans des zones où la rareté des ressources et les conditions climatiques sévères limitent les options pour d'autres formes de subsistance, La migration saisonnière est intégrée comme pratique gestionnaire dans l'activité de l'élevage ${ }^{38}$. La majorité des gens dépendent de l'environnement pour vivre. La terre agricole est un bien recherché tant par les communautés sédentaires que par les agro-pasteurs pour la culture de rente et la culture vivrière. Les

36. Homer-Dixon T., 1998, op. cit.

37. Ibid., p. 207.

38. Saeed A. et al, Study to Assess Factors Contributing to Conflict among Pastoralists, AgroPastoralists and Sedentary Farmers: Stage I Report, Egis Bceom International and Sudan Ministry of Animal Resources and Fisheries, 2009a, p.46. 
pâturages sont essentiels pour les pasteurs mais aussi pour les agriculteurs installés qui possèdent du bétail. Ceux qui sont installés peuvent être soit de petits exploitants soit de grands propriétaires pratiquant l'agriculture mécanisée. L'eau est utilisée pour le bétail et l'horticulture, tandis que l'agriculture est essentiellement pluviale ${ }^{39}$.

Les pasteurs et agro-pasteurs pratiquent le pâturage de saison sèche et le pâturage de saison humide, généralement en suivant un axe nord-sud (durant la saison sèche) et sud-nord (durant la saison des pluies). Certains itinéraires de migration du bétail, qui passent par des zones agricoles et forestières, et traversent les frontières étatiques, sont vieilles de centaines d'années, tandis que d'autres ont été établies pendant la période coloniale. Les routes migratoires font partie intégrante de l'identité pastorale et de son mode de vie. "Il ne s'agit pas seulement d'itinéraires de transit de $\mathrm{A}$ à $\mathrm{B}$ mais de routes qui doivent satisfaire toutes les composantes de la vie sociale des éleveurs, comme le commerce, les cérémonies et les engagements familiaux 40 ». En ce sens, les impacts des changements dans ces routes migratoires sont plus importants qu'une simple menace pour la subsistance, ils affectent d'autres aspects du bien-être.

\section{L'bistoire politique et le contexte}

Une analyse des conflits agriculteur-éleveurs dans le Kordofan méridional ne peut par ailleurs ignorer la réalité historique et ethno-politique et le contexte post-conflit politique, qui demeurent des facteurs clé dans les défis rencontrés ${ }^{41}$.

Le Kordofan Sud, juste au nord de la nouvelle frontière soudanaise avec le Sud-Soudan, englobe le groupe ethnique des Nuba, concentré autour de la région des monts Nouba, qui représente environ $75 \%$ de la population de l'État. Les Nuba pratiquent généralement l'agriculture et sont chrétiens, musulmans ou animistes. Environ 23 \% de la population est arabe Baggara, comprenant les Messiriya, Hawazma et d'autres tribus, qui pratiquent en majorité le pastoralisme et suivent le plus souvent l'Islamisme. Il y a aussi un petit nombre de marchands arabes, appelés Jellaba ${ }^{42}$. Avant la seconde guerre

39. Siddig E. F. A., El-Harizi K., Prato B., Managing Conflict Over Natural Resources in Greater Kordofan, Sudan: Some Recurrent Patterns and Governance Implications, IFPRI Discussion Paper 00711, Washington DC, International Food Policy Research Institute, août 2007, p. 11.

40. El Hassan B. A., Birch I., "Securing Pastoralism in East and West Africa: Protecting and Promoting Livestock Mobility : Sudan Desk Review”, Institut international pour l'environnement et le développement (IIED), 2008, http://pubs.iied.org/pdfs/G03032.pdf (Page consultée le 15 février 2011).

41. Siddig et al, op. cit. ; Large J., Suleiman El-Basha E., A bitter harvest and grounds for reform: the Nuba Mountains, conflicted land and transitional Sudan, Working Paper, Berghof Peace Support, Berlin, juillet 2010.

42. Suliman M., “The Nuba Mountains of Sudan: resource access, violent conflict, and identity", in Buckles D. (ed), Cultivating peace: conflict and collaboration in natural resource management, IDRC, World Bank Institute, Ottawa, 1999. 
civile soudanaise, qui a commencé en 1983, les Nuba et Baggara vivaient, depuis plus de 200 ans, dans une paix relative autour des et dans les monts Nouba où ils ont développé des moyens de partage des ressources de la région ${ }^{43}$.

Toutefois, pendant la deuxième guerre civile qui a duré plus de 20 ans et s'est achevée en 2005, les Nuba ont participé aux combats aux côtés de ceux qui étaient nommés à l'époque l'Armée de Libération du peuple soudanais du sud (APLS). En réaction, le gouvernement soudanais - en utilisant une idéologie panarabe et islamique - a armé les Baggara contre les Nuba 44. L'Accord de paix global de 2005 (CPA) annonçait une accalmie dans les relations entre les agriculteurs Nuba et les éleveurs Baggara, ainsi qu'une probable sécession du Sud Soudan. Cependant, peu avant la sécession en Juillet 2011, des violences ont éclaté dans le Kordofan méridional entre les forces gouvernementales soudanaises et le Mouvement de Libération, nouvellement intitulé Peuple du Soudan (SPLM) ${ }^{45}$, apparemment après que les Forces armées soudanaises eurent présenté un ultimatum au SPLM le sommant de se retirer du Kordofan Sud, au sud de la frontière nord-sud avec le futur Sud-Soudan ${ }^{46}$. Au moment de la rédaction du présent article, les médias et les agences d'aide humanitaire sont interdits dans la région mais des combats de faible intensité semblent se poursuivre entre les rebelles et le gouvernement ${ }^{47}$.

À la lumière de la sécession du Sud-Soudan et des évolutions récentes dans le Kordofan méridional, il est très probable que les politiques étatiques et le débat politique subiront des transformations et il n'est pas déraisonnable de penser que les politiques publiques et le débat politique seront influencés par la rhétorique pro-arabe du Nord. Cependant, le gouvernement continuera à composer avec les défis du changement climatique, et il sera sans doute nécessaire de gérer la rivalité entre les éleveurs et les agriculteurs autour de l'appropriation des ressources - et ceci non seulement dans le Kordofan méridional, mais aussi dans d'autres États.

43. Suliman M., op. cit., cité par Bronkhorst S., Climate change and conflict: lessons for conflict resolution from the southern Sabel of Sudan, Policy and practice report, Durban, African Centre for the Constructive Resolution of Disputes (ACCORD), 2011.

44. Ibid., p. 214 ; Keen D., Complex Emergencies, Cambridge, Polity Press, 2008.

45. Mission des Nations Unies au Soudan (MINUS), “Thousands displaced due to Southern Kordofan fighting shelter near UNMIS”, Featured News, Khartoum, 8 juin 2011, http://unmis.unmissions.org/Default.aspx?tabid=600\&ctl=Details\&mid=697\&ItemID=1394 5 (Page consultée le 20 décembre 2011).

46. New York Times, 30 mai 2011, cité par Rottenburg R., Komey G., Ille E., The genesis of recurring wars in Sudan: rethinking the violent conflicts in Nuba Mountains/Southern Kordofan, University of Halle, Germany, octobre 2011.

47. Reuters, "Sudan and southern rebels clash in oil border state", Khartoum, 14 janvier 2012. Pour un récent examen critique de la résurgence du conflit armé dans le sud du Kordofan, qui comprend une chronologie des événements voir Rottenburg et al, 2011, op. cit. 
Traditionnellement, la rareté des ressources et la compétition intense entre et au sein des groupes de pasteurs, d'agro-pasteurs et d'agriculteur autour des terres et de l'eau ont été atténuées par les migrations saisonnières, la résolution traditionnelle des conflits et les mécanismes de gestion de l'environnement ${ }^{48}$. Récemment, la rivalité dans le Kordofan Sud a été exacerbée en raison des changements naturels et anthropiques qu'a subi l'environnement ${ }^{49}$ mais aussi à cause des développements politiques susmentionnés.

Nonobstant les facteurs politiques, une typologie des conflits liés aux ressources se dessine ${ }^{50}$, confirmée par d'autres études menées principalement par des agences d'aide et des ONG présentes dans la région ${ }^{51}$. La plupart des conflits se produisent le long des voies de migration du bétail ou près des villages des fermiers, lorsque le bétail entre dans les terres agricoles ou lorsque les agriculteurs cultivent des terres traditionnellement dévolues au pastoralisme. Les conflits résultent souvent du déplacement des routes migratoires traditionnelles et historiques dans la mesure où ces routes ont été souvent détruites ou modifiées, notamment par l'introduction de grands projets d'agriculture mécanisée et en raison de la guerre civile. Les routes migratoires ou le calendrier de la migration peuvent aussi changer selon les conditions météorologiques et les précipitations, ce qui contribue aussi à l'émergence de conflits. Des conflits sont également enregistrés entre les groupes pastoraux et les propriétaires de grandes exploitations mécanisées, étant donné que les itinéraires du bétail et les ressources en eau essentielles pour le bétail en mouvement ont été intégrés dans les fermes commerciales. Les conflits sur l'eau pour la consommation animale et humaine sont très répandus autour de trous d'eau (hafirs), creusés par l'homme et traditionnellement destinés au bétail. Or, certains ont été clôturés par les agriculteurs pour d'autres activités ou ne sont pas en mesure de supporter la demande des populations, en particulier durant les périodes de sécheresse ${ }^{52}$. Les types de conflits enregistrés varient entre litige

48. Saeed A. et al., 2009a, op. cit. ; PNUD, op.cit.

49. PNUE, op. cit.; PNUD, op. cit. ; Saeed A. et al., 2009a, op. cit. ; Saeed A. et al, Study to Assess Factors Contributing to Conflict among Pastoralists, Agro-Pastoralists and Sedentary Farmers: Stage II Report, Egis Bceom International and Sudan Ministry of Animal Resources and Fisheries, 2009b.

50. Voir Egeimi O., Mahmood M. A., Ahmed, A. S., “Conflict Transformation: Pastoralists and Settled Farmers”, Compass Magazine, 2003, pp. 22-23 ; El Hassan B. A., Birch, I., op. cit. ;. Suliman M., op. cit. ; Egemi O., Pantuliano S., Land Tenure and the Political Economy of Local Level Conflict in the Sudan: Quest for Social Peace and Sustainable Community Governance, Proceedings of the 7th OSSREA Congress, Khartoum, 15-19 December 2002, publié en 2003 ; Large J., Suleiman El-Basha E., op. cit. ; Bronkhorst S., op. cit.

51. Siddig E. F. A., El-Harizi K., Prato B., Managing Conflict Over Natural Resources in Greater Kordofan, Sudan: Some Recurrent Patterns and Governance Implications, IFPRI Discussion Paper 00711, Washington DC, Institut international de recherche sur les politiques alimentaires, août 2007, http://ageconsearch.umn.edu/bitstream/42402/2/ifpridp00711.pdf (Page consultée le 8 février 2011).

52. Siddig E. F. A., El-Harizi, K., Prato B., op. cit. ; Bradbury M., Ryle J. Medley M., SansculotteGreenidge K., Local peace processes in Sudan: a baseline study, London, Rift Valley Institute 
et combat, entre recours à la médiation formelle, pour les groupes y ayant accès, et violence collective. Certains kordofaniens du sud entrent même en conflit avec d'autres dans les États voisins. Une violence collective insistante est principalement enregistrée dans le Kordofan méridional en raison des tensions entre Nuba et Baggara. Différents exemples de litiges et/ou de conflits violents peuvent être cités 53 :

- Les villages de Babanusa et Al Salam, où la rivalité autour de l'eau est une source de conflit car, sur le parcours migratoire, les agriculteurs et les éleveurs sont en compétition pour sa consommation pour l'agriculture, pour le bétail et pour eux-mêmes ${ }^{54}$.

- Heiban, où l'usage de la terre constitue un facteur de conflit entre les tribus de Uturu, Tira, Lira, Shawaya et Heiban ${ }^{55}$.

- Kordofan Sud où on observe des conflits entre les tribus Birgid Awlad Hilal et les Dar Bakhota, de la tribu arabisée Hawazma ${ }^{56}$.

- Conflits sur l'eau et la terre entre les Shanabla, nomades chameliers sans terre du Nord-Kordofan, et les Nuba 57.

- Nord-Kordofan où les conflits sur l'usage de la terre pour le pâturage et l'agriculture, ainsi que sur l'eau impliquent les tribus Baggara du Kordofan Sud : des tensions surviennent chaque année entre le groupe tribal Gawamaa de Gagrur, et les éleveurs de Sebeihat et Baggara ${ }^{58}$.

La typologie et les exemples cités ci-dessus montrent que la relation entre les migrations pastorales et l'émergence de conflits présente des similitudes avec la bibliographie générale relative aux relations entre les migrations et les conflits, tout en mettant l'accent sur la rareté : les agriculteurs et les éleveurs dépendent pour leur subsistance d'un ensemble de « ressources essen-

and Department for International Development (DFID), 2006, http://www.sudanarchive.net/cgi-bin/sudan?a=pdf\&d=Dunepd250.1\&dl=1 (Page consultée le 21 juin 2011).

53. La société civile ou les organisations internationales sont au courant de différends et de violences à petite échelle qui restent souvent non enregistrés. De conflits tribaux de grande ampleur qui impliquent de la violence sont souvent le résultat de facteurs ethniques et politiques exacerbés par la rareté des ressources. Pour d'autres exemples de conflits voir PNUD 2006, op. cit. ; Saeed A. et al., op. cit. ; Saeed A. et al, 2009b, op. cit. ; Siddig et al, op. cit.

54. SECS, 2010 cité par Bronkhorst, 2011, op. cit.

55. Bronkhorst, 2011, op. cit.

56. Ibid.

57. Fonds des Nations Unies pour l'enfance (UNICEF), Evaluation: grassroots peacebuilding project, Khartoum, Rights Protection and Peace-Building section, 30 septembre 2004 http://workplan.unsudanig.org/mande/reports/docs/evaluations/Sudan_2004_006_Grassro ots_Peace_Building.pdf (Page consultée 20 décembre 2011).

58. Egeimi O., Mahmood M. A., Ahmed A. S., "Conflict Transformation: Pastoralists and Settled Farmers", Compass Magazine, 2003. 
tielles » 59 mais les évolutions naturelles ou anthropiques peuvent rendre ces ressources rares, augmentant par là la probabilité de conflit. Dans le Kordofan méridional, la typologie permet de soutenir la thèse de Reuveny 60 relative aux canaux par lesquels des conflits peuvent surgir en raison d'une migration, tels que la compétition pour des ressources rares, aggravé par les tensions ethniques et religieuses, la méfiance entre groupes et d'autres lignes de clivages socio-économiques ${ }^{61}$.

\section{Quelle est la cause de la rareté des ressources?}

S'appuyant sur les travaux de Homer-Dixon et McLeman ${ }^{62}$, il est instructif de se demander ce qui cause les raretés et, en se rappelant les trois façons pour une ressource de devenir rare, d'identifier de quel type de raretés il s'agit. Dans le Kordofan Sud, la dégradation de l'environnement, liée à la désertification, au surpâturage et à la déforestation, est un problème clé qui provoque une diminution de la qualité et de la disponibilité des ressources ${ }^{63}$. La modification des routes migratoires, comme conséquence de la guerre civile ou parce que les territoires d'accueil sont confrontés à la sécheresse ou ont été dégradées, signifie que les ressources disponibles dans d'autres territoires - qui doivent alors accueillir des personnes supplémentaires et leur bétail - sont touchées à leur tour ${ }^{64}$.

D’autres raretés sont souvent du troisième type et de nature structurelle, où les ressources deviennent moins disponibles pour certains groupes ${ }^{65}$ qui, ayant été exclus, causent des raretés ailleurs puisqu'ils ont besoin d'occuper de nouvelles terres et sources d'eau. On mettra l'accent ci-après sur le fait que ces pénuries sont souvent le résultat de politiques élaborées par l'État et au niveau national, et des faiblesses inhérentes à ces politiques, à la gouvernance et aux institutions à tous les niveaux. La conclusion portera sur les implications de la considération du pastoralisme comme une forme proactive d'adaptation au changement climatique.

\section{Terre, politique agricole mécanisée et législation}

Le gouvernement national post-colonial a introduit, dans les années 1970 et 1980 , une série de lois ${ }^{66}$ (dont beaucoup portent sur les questions foncières)

59. McLeman R., op. cit.

60. Reuveny, R. op. cit.

61. Large J., Soliman El-Basha E., op. cit.

62. Homer-Dixon T., 1998, op. cit. ; McLeman R., op. cit.

63. Saeed A., 2009a, op. cit. ; Bronkhorst S., 2011, op. cit. ; Programme des Nation Unies pour le Développement (PNUD), Pastoral production systems in Southern Kordofan, Khartoum, 2006.

64. Egemi O., Pantuliano S., Land Tenure and the Political Economy of Local Level Conflict in the Sudan: Quest for Social Peace and Sustainable Community Governance, Proceedings of the 7th OSSREA Congress, Khartoum, 15-19 décembre 2002, publié en 2003.

65. Homer-Dixon T., 1998, op. cit. 
et de politiques qui ont provoqué une pénurie de terres disponibles pour les pâturages et l'agriculture et une raréfaction des ressources en eau. Au delà de la variabilité climatique, ces politiques et lois ont eu un effet dévastateur sur les Nuba et les Baggara, détruisant les relations symbiotiques traditionnelles entre les deux groupes qui étaient basées sur le partage et la protection des ressources ${ }^{67}$. En provoquant des déplacements, la raréfaction des terres, et en minant la propriété coutumière des terres ${ }^{68}$, ces initiatives ont également amené, comme indiqué plus haut, un grand nombre de Kordofaniens du sud à rejoindre le SPLM dans la guerre civile ${ }^{69}$.

En ce qui concerne la législation foncière, la loi de 1970 sur les terres non enregistrées 70 déclara tous les biens non enregistrés (c'est à dire des terres communales) comme propriété publique, abolissant ainsi les droits coutumiers à la terre et ouvrant la voie à des saisies de terres en faveur des investisseurs privés ${ }^{71}$. La loi sur les transactions civiles de 1984 (qui a abrogé la loi de 1970), rend plus facile pour les élites l'obtention des terres au détriment des populations rurales ${ }^{72}$. Bien que la loi déclare que les droits d'usufruit enregistré à la terre sont égaux à ceux de la propriété enregistrée, une révision de la loi en 1991 interdit la reconnaissance des droits fonciers coutumiers par les tribunaux ${ }^{73}$. Par ailleurs, la loi de 1974 sur l'intrusion criminelle a augmenté le pouvoir des locataires d'empêcher l'accès des agriculteurs et des éleveurs aux terres antérieurement considérées comme propriété collective ${ }^{74}$. La loi sur l'investissement et la propriété foncière de 1990 a par ailleurs conduit à l'expropriation de certaines zones tribales traditionnelles transformées en propriété d'investisseurs privés ${ }^{75}$.

66. Pour une chronologie détaillée de la législation qui a affecté l'accès à la terre pour les pasteurs et les agriculteurs entre 1970 et 1990 voir El Hassan O., Birch S., op. cit.

67. Rottenburg R., "Introduction", in Nomadic-sedentary relations and failing state institutions in Darfur and Kordofan (Sudan), Orientwissenschaftliches Zentrum, Martin-LutherUniversität Halle-Wittenberg, 2008, pp. vii-ix.

68. Voir Siddig E. F. A., El-Harizi K., Prato B., op. cit. ; Large J., Suleiman El-Basha E. op. cit. ; Egeimi O., Pantuliano S., op. cit. ; Saeed A. et al., 2009a, op. cit. ; Saeed A. et al., 2009b, op. cit. ; Bronkhorst S. et al., op. cit.

69. Pantuliano S., "The land question: Sudan's peace nemesis", Humanitarian Policy Groups, Institut du développement outre-mer, mars 2007, http://www.internaldisplacement.org/8025708F004CE90B/\%28httpDocuments\%29/C4141146535A14A9C125 73AE0059266A/\$file/Land+issues+in+Sudan+-+SSRC+briefing.pdf (Page consultée le 4 juin 2011) ; Keen D., The Benefits of Famine: A Political Economy of Famine and Relief in Southwestern Sudan, 1983-1989, Princeton, Princeton University Press, 1994.

70. À l'époque, presque toutes les terres dans le Kordofan méridional étaient détenues par la communauté. Certains estiment que dans l'ensemble du Soudan unifié seulement $10 \%$ des terres a été inspecté et enregistré (USAID, 2010).

71. El Hassan B. A., Birch, I. "Securing Pastoralism in East and West Africa: Protecting and Promoting Livestock Mobility: Sudan Desk Review”, International Institute for Environment and Development (IIED), 2008.

72. Pantuliano, op. cit.

73. Keen D., 1994, op. cit.

74. El Hassan B. A., Birch I., op. cit.

75. Large J., Soliman El-Basha E., op. cit., Egeimi O., Pantuliano S., op. cit. 
En ce qui concerne l'agenda «pro-croissance », soutenu par la Banque mondiale, la loi susmentionnée (ainsi que la loi sur la corporation agricole mécanisée de 1968 et la loi sur l'encouragement des investissements de 1990) a permis la formation de grandes exploitations mécanisées sur des terres fertiles, antérieurement communales ou tribales, du Kordofan Sud, dans les fertiles monts Nouba et dans certains autres États, à partir de la plupart des fermes appartenant à des hommes d'affaires du Nord ou de Khartoum ${ }^{76}$. Il est estimé qu'en vertu de cette politique de développement de la superficie totale de l'agriculture mécanisée, celle-ci a été multipliée par quinze entre 1970 et 200577.

Suite à la législation et aux politiques relatives à l'agriculture mécanisée dans l'État de Kordofan méridional et les monts Nouba, en particulier, les expropriations étaient à l'ordre du jour et, là où la terre n'a pas été reprise par les exploitations mécanisées, elle est devenue la propriété d'autre acquéreurs ${ }^{78}$. Par ailleurs, le gouvernement pourrait utiliser la force pour protéger les terres gouvernementales, ce qui a permis aux détenteurs de baux de faire respecter l'exclusion des éleveurs et des agriculteurs sur les terres nouvellement acquise ${ }^{79}$. Cette situation a créé de graves pénuries pour les pâturages et l'agriculture, plus particulièrement pour les agro-pasteurs qui se sont vus interdire l'accès à leurs terres d'origine (dars). En outre, les droits de propriété traditionnels ont été en grande partie abolis et peu de droits coutumiers sont maintenus sur les terres laissées aux éleveurs - avec un impact sur l'utilité et la rentabilité de ces terres ${ }^{80}$. Les exploitations mécanisées ont également fait disparaître ou bloquer les routes migratoires traditionnelles de Baggara et les ressources présentes le long de ces itinéraires (telles que l'eau et les terres de pâturage nécessaires pour le bétail des éleveurs). Cela signifie que les éleveurs en mouvement n'avaient pas d'autre choix que d'empiéter avec leur bétail sur les fermes mécanisées ou sur de petites exploitations, tandis que, dans le même temps, ceux qui sont restés sans terre, en raison de la législation, ont commencé à cultiver les terres traditionnellement réservées au pâturage ${ }^{81}$. Selon Ayoub cette situation « demeure une source majeure de mécontentement et de conflits, renforçant le sentiment d'abandon, de marginalisation et de répression sociale, pendant que le blocage des itinéraires nomades, des points d'eau et de pâturage, a favorisé la pratique d'accaparement des terres et la création de larges groupes sans terre 82 ».

76. Saeed A., 2009a, op. cit. ; Egeimi O., Puntaliano S., op. cit.; Ayoub M., "Land and conflict in Sudan”, Conciliation Resources, London. http://www.c-r.org/ourwork/accord/sudan/Land-conflict.php (Page consultée le 20 juin 2011).

77. Ayoub M., op. cit.

78. Large J., Soliman El-Basha E., op. cit.; Pantuliano S., op. cit.

79. El Hassan B.A., Birch I., op. cit.

80. Ayoub M., op. cit.; PNUD, op.cit. ; Pantuliano S., op. cit.

81. Saeed A., 2009a, op. cit. ; Egeimi O., Puntaliano S., op. cit.

82. Ayoub M., op. cit. 
Le Protocole de 2004 entre le gouvernement du Soudan et le SPLM sur la résolution des conflits dans les États de Kordofan méridional / monts Nouba et du Nil Bleu ${ }^{83}$, précédant le CPA, prévoit des commissions foncières dans le sud du Kordofan et le Nil Bleu pour aborder les questions de propriété foncière. Bien que ces dispositions et celles contenues dans le CPA de 2005 soient faibles, elles ont permis des changements juridiques et politiques dans la façon dont le gouvernement a traité les questions de droits fonciers ${ }^{84}$. Bien que des progrès importants aient été réalisés dans le Sud-Soudan, très peu de progrès ont été enregistrés au Kordofan Sud et dans l'État de Nil bleu. Les gouvernements des États membres ont omis de reconnaître et de légaliser les droits coutumiers sur la terre, ou d'établir des commissions foncières (dont le but était, en partie, de corriger les injustices politiques des années 1960). Certains prétendent que cela illustre un manque évident de volonté politique, marquée par l'allocation continue de terres coutumières à des investisseurs extérieurs dans le sud du Kordofan ${ }^{85}$.

Cela dit, des mesures semblent avoir été prises par le gouvernement pour prendre en considération les besoins des éleveurs et les itinéraires pour le bétail ${ }^{86}$, par exemple par le gel, le repositionnement et la réaffectation des terrains en vue de réduire la pénurie en terres de pâturage ${ }^{87}$. D'autres interventions concernent la législation pour définir des itinéraires pour le bétail. Cependant, il y a un fort déficit d'application 88 ou de mise en œuvre des politiques malgré quelques décrets présidentiels sur le sujet ${ }^{89}$. Au niveau de l'État, toute mise en ouvre de la politique de gestion des terres est gravement compromise par un manque majeur de données et d'informations sur le système pastoral, l'utilisation du sol et les changements d'utilisation du sol, la taille de la population humaine et animale. Même la documentation sur les anciens projets environnementaux et agricoles manque ${ }^{90}$. Par exemple, selon Saeed ${ }^{91}$, le dernier recensement du cheptel date de 1976, la dernière enquête sur le revenu des ménages et les dépenses est de 1978 et il est presque impossible d'estimer la taille de la population humaine et animale. De plus, il n'y aurait aucune surveillance par le gouvernement de l'utilisation des sols et des changements survenus. Si les ONG et les organisations internationales font des compilations de données, elles communiquent peu avec le gouvernement. En outre, les itinéraires du bétail ne sont pas définis et sont en constante évolu-

83. Signé à Naivasha, Kenya, le 26 mai 2004. http://www.iss.co.za/af/profiles/sudan/protnilemay04.pdf

84. Alden Wily L., "Making Peace Impossible? Failure to honour the land obligations of the Comprehensive Peace Agreement in Central Sudan", Chr Michelsen Institute, Resource Paper, Oslo, September 2010.

85. Ibid.

86. Bradbury M., Ryle J. Medley M., Sansculotte-Greenidge K., op. cit.

87. PNUD, op. cit.

88. Saeed A., 2009a, op. cit.

89. Babiker M., Entretien téléphonique avec l'auteur, le 9 août 2011.

90. PNUD, op. cit.

91. Saeed A., 2009a, op. cit. 
tion en raison des changements environnementaux naturels et autres ${ }^{92}$. Enfin, les problèmes que rencontre l'administration foncière du gouvernement au niveau local, font que, nonobstant la corruption, la documentation, les données du cadastre et l'information sont souvent perdues, ce qui rend la gestion et l'attribution des terres, ou la sécurisation des droits fonciers hautement problématique ${ }^{93}$.

\section{La gestion traditionnelle de l'environnement et des conflits}

Pour ce qui concerne l'adaptation face à la rareté découlant du changement climatique et de la variabilité du climat, le rôle des institutions locales en milieu rural est crucial. «Non seulement les institutions influent sur la façon dont les ménages sont affectés par les impacts du changement climatique, mais aussi elles forgent la capacité des ménages à répondre à ces impacts, à poursuivre des pratiques différentes d'adaptation, et à gérer les interventions extérieures dans le contexte de l'adaptation. L'accès des ménages et de différents groupes sociaux aux institutions et aux ressources institutionnellement allouées est un facteur déterminant dans leur capacité à s'adapter avec succès 94 ». En conséquence, pour une adaptation réussie, il sera crucial d'établir et de renforcer les institutions afin de gérer les ressources plus équitablement, de faire face à leur rareté et de gérer et prévenir conflits, en particulier dans le cas du pastoralisme.

Cependant, alors que les institutions pourraient aussi aborder et atténuer ces pénuries et prévenir les conflits, elles peuvent également être affectées par le contexte politique. Rottenburg affirme qu'au Kordofan Sud « les conflits entre agriculteurs et éleveurs ... [sont] liés à l'échec des institutions étatiques moderne, aux politiques nationales mal conduites, et aux stratégies de développement imprécis poursuivies par l'État qui néglige les intérêts et les priorités des communautés d'agriculteurs et des éleveurs... 95 ». De tels conflits ne devraient pas être appelés « conflits de ressources ", " conflits pour le pétrole », " conflits ethniques », " conflits raciaux », " conflits d'intérêts entre l'agriculture et l'élevage » ou « conflits climatiques ». L'étiquette devrait plutôt être «conflits causés par une défaillance institutionnelle».

Dans le Kordofan méridional, ce type d'institution, traditionnellement celle de l'administration indigène (NA) incluant les autorités traditionnelles de cheikhs, omdas et Nazirs, a joué un rôle crucial dans la gestion des ressources naturelles, la mobilité du bétail, la séparation des pâturages et des

92. Ibid.

93. Pantuliano S., op. cit.

94. Agrawal A., "The Role of Local Institutions in Adaptation to Climate Change", Social Dimensions of Climate Change, Social Development Department, Washington DC, Banque Mondiale, 5-6 mars 2008.

95. Rottenburg R., op. cit., p. ix. 
espaces cultivables, ainsi que dans la résolution des conflits entre tribus. Cette institution traditionnelle était relativement bien placée pour gérer et traiter la rareté des ressources et les conflits qui peuvent surgir entre et au sein des tribus ${ }^{96}$ et sans doute, si son pouvoir avait été renforcé, elle aurait même pu atténuer certains des dommages causés par la législation foncière et les politiques introduites par le gouvernement central. Selon El Hassan et Birch, « des ordonnances locales prévoyaient le moment et la direction des mouvements pastoraux le long des itinéraires du bétail, l'ouverture et la fermeture des points d'eau et la date limite pour la récolte après quoi le bétail pouvait entrer dans les champs et paître les résidus de récolte 97 ». L'agriculture était interdite le long des itinéraires, et des accords ont été périodiquement conclus entre les groupes, afin de prévenir les conflits ${ }^{98}$. Un autre aspect important de l'administration indigène a été d'être largement responsable de la médiation coutumière (juddiya) ${ }^{99}$. Celle-ci a joué un rôle clé dans la gestion des raretés et dans la prévention des conflits autour des ressources rares au niveau communal. Elle est même utilisée pour résoudre les graves conflits inter-tribaux au Soudan 100 .

Cependant, une autre évolution politique, dans les années 1970, a conduit à l'abolition de l'administration indigène. Bien que rétablie dans les années 1980, elle est en proie à une multitude de défis, y compris un manque de pouvoir et de légitimité, la montée de la militarisation des jeunes, l'ingérence politique et un manque de capacités techniques pour faire face aux récents défis écologiques et politiques ${ }^{101 .}$

Par ailleurs, comme des médiateurs ont été choisis parmi les administrateurs indigènes après l'abolition de leur administration d'origine, ceci a laissé un grand vide, notamment en termes de gestion des ressources et de résolution des conflits. Bien que le gouvernement soudanais ait adopté des mesures pour remplacer le fonctionnement de l'administration indigène, il semble y avoir un large consensus autour du fait que cette opération n’a pas été un grand succès, principalement en raison de la faiblesse des capacités d'action au niveau local, d'un manque de coordination et des chevauchements entre les différents secteurs d'action publique impliqués dans les affaires locales ${ }^{102}$. Un inconvénient

96. Bronkhorst S., op. cit.

97. El Hassan B.A., Birch I., op. cit., p. 7

98. Wadi E.-T. I., El Hillo M., Hadi M.B., Resource-based conflict and mechanism of conflict resolution in North Kordofan, Gedarif and Blue Nile States, Khartoum, Sudan Environmental Conservation Society, 2005, p. 35.

99. Pour une discussion détaillée de la pratique de judiyya comme forme de gestion des ressources au Soudan, voir Bronkhorst S., Resource Scarcity and Endogenous Systems of Conflict Management in Sudan: Customary Mediation (Judiyya), Traditional Conflict Resolution in Africa, Durban, ACCORD, 2012, (à paraître).

100.Ibid.

101.Wadi E.-T, I. El Hillo M., Hadi, M.B., op. cit.

102.Elhussein, A.M., “The revival of 'native administration' in the Sudan: a pragmatic view”, Public Administration and Development, vol. 9, 1989, pp. 437-446. 
majeur a été la gestion des affaires locales, qui n'a été attribuée à personne. Il est probable que ces difficultés ont contribué à une restauration de l'administration indigène, mais déjà, le mal était fait, et les administrateurs indigènes n'ont pas obtenu la même légitimité et le même pouvoir, et donc la capacité à gérer les ressources rares, comme ils le faisaient dans le passé ${ }^{103}$. Il est également vrai que le rôle le plus puissant (celui du Nazir) n'a pas été rétabli - il y a là une forme de manipulation des pouvoirs locaux par le gouvernement afin de conserver le soutien autour de ses politiques pro-Islam ${ }^{104}$.

Bien qu'il y ait des preuves prometteuses que les autorités traditionnelles sont renforcées et réintroduites au Soudan 105 et que dans certains cas les gouvernements des États ont délégué des pouvoirs aux administrateurs indigènes pour gérer les ressources, l'ingérence politique reste un défi important. Selon Bradbury et al, cette interférence résulte des "négociations entre pouvoirs locaux et nationaux 106 ». La manipulation par le gouvernement des institutions et autorités tribales érode donc davantage sa légitimité et le rôle qu'il peut jouer dans la gestion des ressources et la résolution des conflits entre différents groupes. Depuis juin 2011, un certain nombre d'administrateurs autochtones et de chefs traditionnels auraient été tués dans l'État 107, ce qui souligne la fonction politique des administrateurs indigènes dans l'aprèssécession du Soudan.

\section{Implications pour le pastoralisme dans le Kordofan Sud, et pour le pastora-} lisme comme forme d'adaptation au changement climatique

La section précédente montre que les politiques et la législation gouvernementales ont systématiquement exclu les agriculteurs de la terre et les éleveurs des ressources essentielles à la production pastorale, comme elles ont détruit une institution qui disposait de la capacité de gérer ces ressources et les conflits autour de l'appropriation de ces ressources. Au sein d'un pays par ailleurs aride 108, le Kordofan Sud présente un excellent potentiel agricole mais les politiques et la législation en ont fait bénéficier les plus riches et les plus proches du pouvoir, venant de la région du nord, en privant les agriculteurs et

103. Wadi E.-T. I., El Hillo M., Hadi M. B, op. cit.

104.Al-Hardallu A., El Tayeb S.E., Inter-communal conflict in Sudan: causes, resolution mechanisms and transformation - A case study of the Easter Region, Khartoum, Ahfad University for Women, 2005.

105.Sudan Vision, Nafie to Address the Native Administration Conference in Southern Kordofan, 8 août 2011, http://news.sudanvisiondaily.com/details.html?rsnpid=197673 (Page consultée le 27 décembre 2011); Bronkhorst S., 2011, op. cit.

106.Bradbury M., Ryle J., Medley M., Sansculotte-Greenidge K., op. cit., p. 14

107.Sudan Vision, "SPLM Assassinates Tens of Political, Religious and Tribal Leaderships in South Kordofan State”, 2 janvier http://news.sudanvisiondaily.com/details.html?rsnpid=204311 (Page consultée le 22 janvier 2012).

108. Compte tenu du contexte politique post-colonial, l'ancien Sud-Soudan est exclu de cette analyse. 
les pasteurs de leurs droits et sans leur offrir d'alternatives de subsistance. Alors que les considérations sociales et en matière de développement ont joué un rôle crucial dans les actions gouvernementales, les considérations politiques semblent avoir joué aussi un rôle important - du moins en ce qui concerne les Nuba qui représentaient un groupe non-arabe puissant occupant une région économiquement et politiquement stratégique du Soudan.

Homer-Dixon affirme que ce type de rareté structurelle peut être causé par et générer une "monopolisation des ressources » et une "marginalisation écologique ». La monopolisation des ressources intervient quand des "groupes puissants au sein d'une société reconnaissent qu'une ressource clé devient de plus en plus rare (en raison des pressions liées à la fois l'offre et à la demande) et utilisent leur pouvoir pour détourner en leur faveur le régime régissant l'accès aux ressources. Ce changement impose de graves pénuries structurelles sur les groupes les plus faibles 109 ». Par ailleurs, les effets de la rareté dans une zone poussent aussi les pasteurs et les agriculteurs dans d'autres zones qui peuvent ne pas être optimales pour garantir leurs moyens de subsistance, ce qui renforce davantage le phénomène de rareté. Cette marginalisation écologique se produit « quand un déséquilibre structurel dans la répartition des ressources se joint à la croissance rapide de la population conduisant des personnes à faibles ressources dans les zones écologiquement marginales 110 ». Alors que la croissance de la population n'est pas en cause ici, les éleveurs rencontrent des conditions de rareté artificiellement créées, non seulement sur leurs lieux de vie, mais aussi le long des itinéraires de migration et dans les zones de réception. De surcroit, la désertification et la sur-utilisation de la terre exercent des pressions croissantes transformant certains territoires en zones écologiquement marginalisées.

Si le gouvernement soudanais continue la commercialisation et la privatisation des grandes étendues de terres arables tribales, sans suivre une politique claire qui équilibre les objectifs de l'agriculture commerciale avec le pastoralisme, et les besoins des petits propriétaires, le conflit est susceptible de se poursuivre entre ces acteurs. Pour le pastoralisme en général et, plus particulièrement, pour la réussite du pastoralisme en tant que migration adaptative, les terres pour le pâturage et l'eau pour le bétail sont des ressources cruciales. La privatisation ou le manque de gestion de ces ressources, les rendent inaccessibles pour les éleveurs, ce qui crée de la rareté et induit des confrontations autour de leur appropriation dans d'autres endroits.

Par ailleurs, les institutions sont essentielles pour gérer et négocier les ressources communes et les problèmes de rareté, tant au sein des communautés d'accueil que le long des itinéraires, et peut-être même pour gérer l'accès

109.Homer Dixon T., op. cit., p. 209

110.Ibid. 
négocié à la propriété privée. Dans le contexte de la faiblesse du gouvernement décrite plus haut, l'administration locale paraît peu apte à agir dans ce sens dans le court terme, surtout à la lumière des récentes évolutions politiques. Des succès ont pu être enregistrés uniquement dans les zones où l'administration autochtone fonctionne encore et détient le pouvoir, la légitimité et les moyens pour gérer la rareté des ressources et les conflits. En plus des efforts du gouvernement, certains acteurs non gouvernementaux et des institutions internationales dans le Kordofan-Sud s'appuient sur les mécanismes traditionnels de résolution des conflits et le renforcement des autorités traditionnelles pour gérer les conflits entre pasteurs et agriculteurs ${ }^{111}$. Si les institutions traditionnelles dans d'autres régions de l'État ne sont pas renforcées - soit parce qu'elles ont été irrémédiablement détruites soit parce qu'il n'y a pas la volonté politique - elles doivent être remplacées par des systèmes modernes de gestion des ressources et des conflits. Cependant, jusqu'à ce que le gouvernement de l'État arrive à développer sa capacité à gérer les ressources et les conflits, l'administration autochtone présente une voie possible, du moins provisoire, par laquelle les ressources peuvent être gérées et négociées et les conflits résolus.

La principale question que soulève cet article pour les décideurs politiques soudanais, et pour laquelle il ne propose pas de réponse, consiste à savoir, dans un contexte où les gens sont dépendants de la terre et où le changement et la variabilité climatiques rendent chaque année cette terre inhabitable ou improductive pour une période plus ou moins connue, comment la rareté doit-elle être gérée ? Cette question est cruciale, parce que s'il n'y a pas de volonté politique au Soudan pour promouvoir une forme de migration saisonnière, comme le pastoralisme, en tant que système de survie, comment les régions du nord qui deviendront plus inhospitalières à l'avenir pourront être gérées ? Quel pourrait être l'usage le plus adapté de cette terre fragilisée et comment le gouvernement garantira les moyens de subsistance et trouvera des alternatives plausibles dans ce contexte, si la migration saisonnière n'est pas une option ? Par ailleurs, au vu des conditions imposées par le changement climatique, par d'autres pressions environnementales, par la taille grandissante de la population et par l'insécurité du régime foncier, comment le gouvernement peut équilibrer les avantages de la migration saisonnière avec les objectifs de la croissance économique et la commercialisation du secteur agricole?

Le gouvernement soudanais et l'Etat de Kordofan Sud se trouvent donc face à un dilemme : s'ils considèrent le pastoralisme comme une forme adéquate de migration proactive, et donc d'adaptation dynamique, ils doivent répondre aux enjeux importants qui sont à l'origine des conflits entre agriculteurs et éleveurs. Il ne s'agit pas d'un travail qui peut être abandonné à mi-chemin. Comme le démontre le cas du Kordofan Sud, l'adaptation mise à part, un cadre cohérent de politiques publiques, de législations et de structures admi- 
nistratives de mise en ouvre qui fonctionnent et qui ont la capacité de fonctionner tant au niveau national que local, sont nécessaires. Ce cadre doit concilier les objectifs de croissance économique et de développement, avec le pastoralisme, l'agriculture et les mesures relatives à l'adaptation au changement climatique. Cet article montre que ces objectifs ne peuvent pas être en compétition et faire profiter seuls de petits groupes d'élite. Ils requièrent un niveau de complémentarité pouvant concilier de revendications concurrentes face à des ressources devenues plus rares. Pour que la gestion du pastoralisme et de l'adaptation réussissent, et que le suivi et la gestion des conflits entre pasteurs et agriculteurs réussissent aussi, cela requiert des capacités et des moyens. Sans investissements, visant le renforcement de l'information, des ressources humaines et des compétences en matière d'adaptation et de pastoralisme (à savoir élaboration de politiques, mise en œuvre et gestion), il est peu probable que les politiques d'encouragement du pastoralisme comme une forme d'adaptation réussisse. 